\title{
¿COEXISTENCIA DE CONFIGURACIONES TONALES EN LA VARIEDAD DE ESPAÑOL DE LA GOMERA? ${ }^{1}$
}

\author{
Chaxiraxi Díaz \\ Josefa Dorta \\ Universidad de La Laguna \\ chadiaz@ull.edu.es \\ jdorta@ull.edu.es
}

\begin{abstract}
RESUMEN: AMPER (Atlas Multimedia de Prosodia del Espacio Románico) nace en el año 2002 en Francia con la pretensión de realizar el atlas de prosodia de las lenguas y variedades lingüísticas del ámbito románico, al tiempo que propiciar estudios descriptivos que permitan la comparación entre ellas. AMPERCan se ocupa del estudio geolingüístico de la prosodia del español de Canarias.

El habla de la isla de La Gomera tiene rasgos conservadores en relación con el resto de las Islas Canarias que son, asimismo, afines al español de América debido a la confluencia de determinados factores históricos. En estudios precedentes se ha encontrado un patrón interrogativo ascendente en un corpus de habla formal de esta isla semejante al del castellano que pudiera estar relacionado con el conservadurismo de la isla. La sospecha de que no es así ha motivado el presente estudio en el que describimos las características fonéticas de un conjunto de oraciones, no solo interrogativas sino también declarativas neutras con sentido completo, contrastando corpus distintos con el propósito de comprobar si en los más espontáneos aparecen las mismas configuraciones que en un corpus formal.
\end{abstract}

PALABRAS CLAVE: entonación, acento, prosodia, corpus experimental, corpus espontáneo, español de Canarias.

1. El presente trabajo se enmarca en el proyecto de investigación Estudio comparativo de la entonación y del acento en zonas fronterizas del español (2015-2012017), del Programa estatal de fomento de la investigación científica y técnica de excelencia, subprograma estatal de generación del conocimiento del Ministerio de Economía y Competitividad (FFI2014-52716-P). Este proyecto se vincula, a su vez, a AMPER (Atlas Multimédia Prosodique de l'Espace Roman), coordinado internacionalmente por Michel Contini (Universidad Stendhal-Grenoble 3) y Antonio Romano (Universidad de Turín). El Coordinador del ámbito español es Eugenio Martínez Celdrán (Universidad de Barcelona) y la Vicecoordinadora en España es Josefa Dorta. 


\title{
COEXISTENCE OF TONAL CONFIGURATIONS IN THE VARIETY OF SPANISH OF LA GOMERA ISLAND?
}

\begin{abstract}
AMPER (Atlas Multimedia de Prosodia del Espacio Románico) was set up in France in 2002 with the aim of developing an atlas of prosody of languages and language varieties of Romanesque field, thus enabling descriptive studies to facilitate comparison among them. Specifically, AMPERCan deals with the geolinguistic study of prosody of Spanish in the Canary Islands.

Speech in La Gomera Island presents conservative features as compared to the rest of the Canary Islands, and is also related to Spanish in America due to the confluence of certain historical factors. Previous studies have found an ascending interrogative pattern in a corpus of formal speech from this island that is similar to the Castilian variety; this may explain the conservatism of the Gomeran speech.

However, we suspect that such pattern may be rather due to speech style. In order to test this, the present study describes the phonetic characteristics of a set of sentences, including both interrogative and neutral-declarative sentences with complete sense, and compares different corpuses with the aim of verifying whether the same configurations appear in formal as well as in more spontaneous corpuses.

KEYWORDS: Intonation, stress, prosody, experimental corpus, spontaneus corpus, Spanish Canarian.
\end{abstract}

Recibido: 17/03/2015. Aceptado: 14/05/2015

\section{Introducción}

La Gomera es denominada la isla colombina debido a que fue lugar de avituallamiento de Cristóbal Colón antes de partir al Nuevo Mundo, en 1492. En esa fecha la conquista de las islas por parte de la corona de Castilla prácticamente estaba concluida ${ }^{2}$, máxime en La Gomera que pasa a formar parte del territorio hacia 1450, esto es, desde el primer periodo de los dos que distinguen los historiadores: la Conquista señorial y la realenga ${ }^{3}$. Esta isla y las restantes del Archipiélago se convierten así en la puerta de entrada al océano atlántico, lo que influye claramente en la evolución del idioma resultante en tierras canarias y, asimismo, en la similitud con el español de la América hispana (Corrales y Corbella 2004: 71). Al respecto, en El español de Canarias (Almeida y Díaz Alayón 1988: 13) se afirma que el es-

2. Recordemos que la conquista se inicia en 1405 y concluye en 1496.

3. Las islas del señorío son Lanzarote, Fuerteventura, La Gomera y El Hierro y las del realengo Gran Canaria, Tenerife y La Palma. 
pañol de las islas es "una proyección del que entonces se habla en Andalucía occidental" adaptada a la realidad de Canarias puesto que incorpora elementos del sustrato lingüístico prehispánico, del adstrato occidental ibérico y del español de América. Asimismo, se suele afirmar que la variedad canaria es una de las más estudiadas $^{4}$; no obstante, conviene recordar que el dialectólogo Salvador Caja decía hace años que "no hay dialecto canario en sentido estricto sino múltiples variedades locales, considerables diferencias de isla en isla, enrevesadas isoglosas entrecruzadas sin salir de cada una de ellas" (1990: 101). Uno de los factores decisivos que ha provocado este hecho es, según Morera, “el origen de la población extranjera con la que las distintas islas han estado en contacto a lo largo de su historia y la mayor o menor presión que esta población ha ejercido sobre la gente de cada zona" (2003-2004: 1526-1527). De este modo, La Gomera, junto con las otras islas occidentales, ha tenido más contacto con América que las islas orientales y, por tanto, cabría esperar mayor influencia de Hispanoamérica en los distintos planos del lenguaje. Otro factor, según el mismo autor, es el carácter más o menos conservador de la isla condicionado por su diferente desarrollo social, económico, cultural, etc. Así, las antiguas islas de señorío, esto es, Lanzarote, Fuerteventura, La Gomera y El Hierro se han mantenido siempre más encerradas en sí mismas que las antiguas islas de realengo y, por ello, son aquellas más conservadoras que estas.

A pesar de la abundancia de trabajos sobre el español canario, el de la isla de La Gomera ha sido, en comparación con el del resto de las islas canarias, uno de los menos estudiados ${ }^{5}$ y los trabajos que existen sobre el mismo evidencian el carácter conservador de sus habitantes. Así, por ejemplo, rechazan fenómenos como el yeísmo (Alvar 1968: 71), mantienen en algunas zonas, como en castellano, el pronombre personal vosotros (Almeida y Díaz Alayón 1988: 116), perviven supuestos guanchismos propios de la isla y conservan voces o acepciones desaparecidas del resto del ámbito insular, además de que se advierte una notable influencia de América hasta el punto de que existen palabras desconocidas en el resto de Canarias (Morera 2003-2004: 1527-1529).

4. Ya en el siglo pasado Salvador Caja afirmaba que: "El español hablado en Canarias es, seguramente, la variedad dialectal más estudiada en el último cuarto de siglo" (1990: 96). Hace alusión a la Guía bibliográfica de el español de Canarias (Corrales Zumbado et al. 1998) que reunía entonces 571 títulos.

5. La atención de los investigadores ha estado centrada en la tradición prehispánica del "silbo gomero", Patrimonio de la Humanidad, que continúa viva en algunos núcleos. Es otro ejemplo de que La Gomera es la isla que mejor conserva las tradiciones de los habitantes prehispánicos. Para la caracterización del silbo véase Trujillo 1978. 
La ausencia de estudios sobre el español de La Gomera es más evidente si nos situamos en ámbitos distintos al plano fónico segmental o el léxico. Así, por lo que respecta a la entonación, objeto de interés en este trabajo, la inexistencia de este tipo de estudios es constatable hasta hace pocos años, aunque este hecho es extensible al resto del Archipiélago canario. En efecto, las primeras aproximaciones al análisis de la entonación de la isla colombina nacen en 2007 en el marco del proyecto AMPER al comparar los contornos declarativos (Hernández 2007) e interrogativo (Dorta 2007) del habla femenina rural de La Gomera con los de Gran Canaria. Estos estudios evidencian que, atendiendo al núcleo final, las declarativas se caracterizan por el descenso o final descendente común a otras variedades del español general (Quilis 1993: 428) y a las zonas urbanas de las islas canarias, tal como se ha puesto de manifiesto en estudios posteriores (v. gr. Hernández et al. 2011 y Hernández et al. 2014). Las interrogativas gomeras de la zona rural, en cambio, tienen un movimiento final de ascenso-descenso de la $\mathrm{F}_{0}$ (conocido normalmente como final circunflejo) semejante al de algunas hablas hispanoamericanas (Sosa 1999). Este mismo final se ha registrado comúnmente en las zonas urbanas de Canarias (v. gr. Dorta 2008 y 2013; Hernández et al. 2011; Dorta Ed. 2013) y también en las otras zonas rurales del archipiélago (v. gr. Hernández et al. 2014). Sin embargo, la configuración con final ascendente propia del castellano (Quilis 1993: 429) se ha encontrado en la zona urbana de La Gomera (v. gr. Dorta 2008) por lo que se da un contraste de patrones tonales entre esta zona y la rural con patrón circunflejo (Díaz 2013).

\section{Objetivo e hipótesis}

En el trabajo citado de Dorta (2008), donde se registró una configuración ascendente en las interrogativas de habla urbana de La Gomera, se advertía lo siguiente:

en relación con la configuración ascendente, en un estudio precedente en el que se analizó el mismo corpus experimental (Dorta 2007) emitido por una mujer de La Gomera pero de una zona rural, la configuración final de las interrogativas fue circunfleja como en la mayoría de las islas. Por tanto, en estudios posteriores se verá si el diferente comportamiento tonal que se ha visto en este estudio hay que relacionarlo con la diferencia de zona (rural $v s$. urbana) o si, por el contrario, obedece a características individuales de las informantes o a cualquier otro hecho (2008: 24).

Así, pues, el registro de dos patrones interrogativos, ascendente y circunflejo, en la isla de La Gomera y la posibilidad de que el primero, más ajeno a las hablas canarias y más propio del habla septentrional, sea un rasgo conservador mantenido en esta isla, junto a otros de carácter fónico o léxico como se ha dicho en la intro- 
ducción, ha determinado la cuestión que da título a este trabajo ${ }^{6}$. No obstante, partimos de la hipótesis de que el patrón ascendente pudiera ser más un producto del habla formal por imitación del castellano que un rasgo conservador del habla gomera. Para que nuestra hipótesis tenga mayor fundamento, contrastamos los resultados obtenidos en un corpus formal con otros más espontáneos donde es menos probable la imitación de un patrón entonativo foráneo. El estudio se restringe al ámbito fonético y lo ampliamos a la modalidad declarativa con el propósito de contrastar las dos modalidades para comprobar si los resultados distan sustancialmente de los obtenidos en el español de las otras islas.

\section{Metodología}

\subsection{Puntos de encuesta}

La Gomera es una de las siete Islas Canarias situada en la parte occidental del archipiélago; pertenece al grupo de "las islas menores" (junto con El Hierro y La Palma). Vinculada a la provincia de Santa Cruz de Tenerife, su capital es San Sebastián de La Gomera. Tiene una superficie de $369,76 \mathrm{~km}^{2}$, dividida en los seis municipios que figuran en la figura 1.

Figura 1. Distribución de los municipios de la isla de La Gomera?.

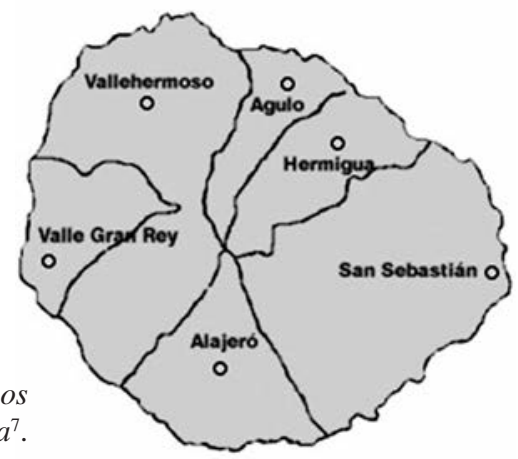

6. En Dorta y Díaz 2014 se aplicó un test de percepción a 34 auditores canarios formado por 66 estímulos sintetizados de tipo prosódico emitidos por informantes procedentes de Canarias y de la Península Ibérica. El objetivo principal de esta investigación era determinar el grado de normalidad que daban los auditores canarios a los estímulos sintetizados obtenidos a partir de oraciones declarativas -corpus de contraste- e interrogativas absolutas con final circunflejo y ascendente. Concretamente, tres de los informantes procedían de La Gomera: uno emitió las interrogativas con tonema circunflejo y dos con tonema ascendente. Una de las conclusiones a las que se llegó es que los auditores canarios percibían de manera similar los dos patrones interrogativos, circunflejo y ascendente. Según las autoras ello se podría explicar tanto porque ambos pertenecen al español, lengua de los auditores, como por el hecho de que los dos se dan en la variedad canaria, hecho probado, al menos, fonéticamente.

7. Agulo, Alajeró, Hermigua, San Sebastián de La Gomera, Vallehermoso y Valle Gran Rey. 
Los puntos de encuesta de este estudio son Valle Gran Rey y San Sebastián: el primero se encuentra al oeste de la isla (extensión de $36,36 \mathrm{~km}^{2}$ ) y el segundo al este $\left(114,47 \mathrm{~km}^{2}\right)$. En el municipio de Valle Gran Rey los informantes encuestados proceden de Guadá, un conjunto de barrios enclavados en la parte alta del barranco. Por su parte, San Sebastián es la capital y puerta de entrada a la isla. En ella se sitúan los principales organismos públicos y el Puerto.

\subsection{Informantes}

Los informantes seleccionados fueron seis. Siguiendo la metodología de AMPER tienen edades que oscilan entre los 18 y los 55 años. Su distribución según las variables nivel de estudios (superiores +; básicos -), procedencia (urbana Ur; rural $\mathrm{Ru}$ ) y sexo (hombre $\mathrm{H}$; mujer M) es la que se muestra en la tabla 1 .

Tabla 1. Distribución de informantes según las variables consideradas

\begin{tabular}{|c|c|c|c|}
\hline \multicolumn{4}{|c|}{ INFORMANTES } \\
\hline \multicolumn{3}{|c|}{ Zona Ur } & \multicolumn{2}{c|}{ Zona Ru } \\
\hline SEXO & NIVEL DE ESTUDIOS & SEXO & NIVEL DE ESTUDIOS \\
\hline $\mathrm{M}$ & - & $\mathrm{M}$ & - \\
\hline $\mathrm{H}$ & - & $\mathrm{H}$ & - \\
\hline $\mathrm{M}$ & + & --- & -- \\
\hline $\mathrm{H}$ & + & --- & -- \\
\hline
\end{tabular}

\subsection{Corpus}

\subsubsection{Corpus fijo o experimental}

El corpus objeto de análisis es, principalmente, el denominado corpus fijo o ad hoc. Este fue emitido en dos modalidades oracionales: declarativas e interrogativas absolutas. Cada una de las frases fue repetida tres veces siguiendo unas estrategias encaminadas a obtener un corpus lo más neutral posible. Está constituido por un conjunto de oraciones de 11 sílabas del tipo SVO: SN (sintagma nominal $)+S V$ (sintagma verbal) + SPrep (sintagma preposicional). En el sintagma nominal y en el preposicional su núcleo lo forma una palabra trisílaba aguda, llana 
y esdrújula. El corpus final está compuesto por un conjunto de 324 oraciones según se muestra en la tabla 2.

Tabla 2. Distribución de las oraciones del corpus fijo

\begin{tabular}{|c|l|c|c|c|c|}
\hline \multicolumn{6}{|c|}{ CORPUS FIJO O EXPERIMENTAL } \\
\hline \multirow{2}{|c|}{ Modalidad } & 3 repeticiones & 6 informantes & Total \\
\hline \multirow{2}{*}{ ORACIONES } & Declarativas & 9 & 27 & 162 & \multirow{2}{*}{324 oraciones } \\
\cline { 2 - 6 } & Interrogativas & 9 & 27 & 162 & \\
\hline
\end{tabular}

La figura 2 ilustra la entonación de una oración SVO con acento inicial y final agudo emitida como declarativa e interrogativa por una mujer de San Sebastián de La Gomera.

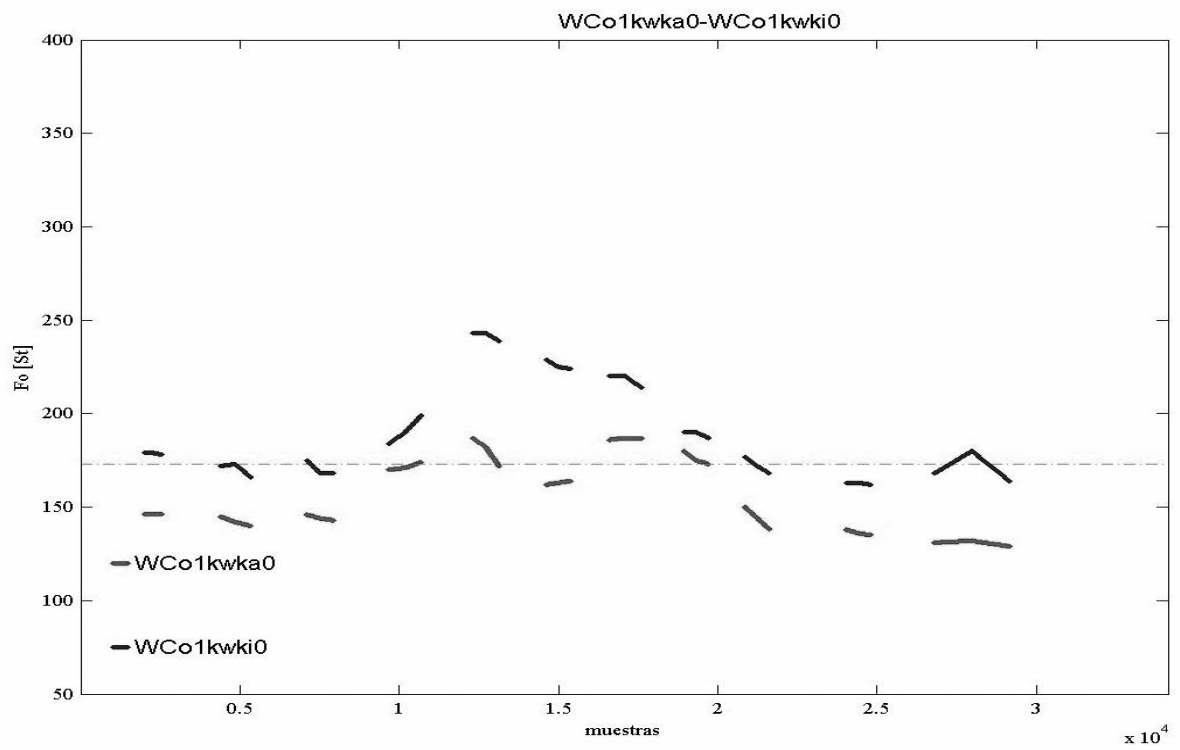

Figura 2. Oración declarativa (inferior) e interrogativa (superior) obtenida con el programa AMPER $2006^{8}$ (corpus ad hoc).

8. Se hablará de este programa en el apartado 3.5. 


\subsubsection{Corpus de contraste}

Con el objetivo de confirmar si el patrón -o patrones- entonativo obtenido en el análisis del corpus experimental es efectivamente el usado en el español hablado en La Gomera y que no está condicionado por la situación más formal que supone la elicitación textual de dicho corpus, hemos analizado otro de contraste más espontáneo siguiendo las directrices del proyecto AMPER, esto es, un corpus inducido (CS) y otro obtenido mediante la técnica map task (CE) ${ }^{9}$.

El corpus definitivo está formado por 170 oraciones declarativas e interrogativas absolutas con esquema acentual llano en su mayoría y, en menor medida, agudo; en ningún caso fue esdrújulo. Véase en tabla 3 la distribución del corpus.

\section{Tabla 3. Distribución del corpus de contraste}

\begin{tabular}{|c|c|c|c|c|}
\hline \multicolumn{5}{|c|}{ CORPUS DE CONTRASTE } \\
\hline \multicolumn{2}{|l|}{ Modalidad } & \multicolumn{2}{|c|}{ Tipo de corpus } & Total \\
\hline \multirow{2}{*}{ Declarativas } & \multirow{2}{*}{71} & Inducido & 52 & \multirow{4}{*}{$\begin{array}{c}170 \\
\text { oraciones }\end{array}$} \\
\hline & & Map task & 19 & \\
\hline \multirow{2}{*}{ Interrogativas } & \multirow{2}{*}{99} & Inducido & 70 & \\
\hline & & Map task & 29 & \\
\hline
\end{tabular}

En relación con la clasificación del corpus de contraste, hemos considerado el relieve tonal de la frase para su delimitación teniendo en cuenta que estudios precedentes - para las dos modalidades oracionales- manifiestan la diferente configuración de las curvas atendiendo a este factor (Torres Álvarez 2000 y Dorta 2000) ${ }^{10}$. Así, en

9. El primer corpus semiespontáneo o inducido (CS) está constituido por frases que se obtienen mediante el planteamiento al informante de cuestiones de uso muy cotidiano. Por ejemplo: "Vas por la calle y no tienes hora. Pasa un señor ¿Cómo preguntarías por la hora que es?” La respuesta que se espera es: “QQué hora es?” u otra similar. El segundo tipo de corpus más espontáneo (CE) se obtiene mediante un sistema de recogida de datos planteado a partir de mapas: el informante y el entrevistador deben salir de un punto geográfico y llegar a un destino determinado. Puesto que los dos mapas son diferentes en algunos puntos, se suscitarán preguntas y respuestas por parte de ambos intervinientes.

10. En el estudio sobre las declarativas del español de Canarias Torres Álvarez (2000) señala que en un $86,28 \%$ las oraciones monocumbres son cortas (de 1 a 7 sílabas) y que en un 92,31\% las oraciones bicumbres son largas (+7 sílabas). Dorta (2000), por su parte, indica que las interrogativas no pronominales son bicumbres cuando presentan más de 6 sílabas. 
las dos modalidades hemos separado las oraciones que tienen un solo pico tonal (cuya extensión va generalmente de 1 a 7 sílabas en declarativas y hasta 6 en interrogativas) de las que presentan más de una cumbre tonal máxima y que superan dichas cifras (más de 7 sílabas en las declarativas y más de 6 sílabas en las interrogativas $)^{11}$. Las figuras 3 y 4 ilustran el comportamiento tonal en el corpus semiespontáneo o inducido.

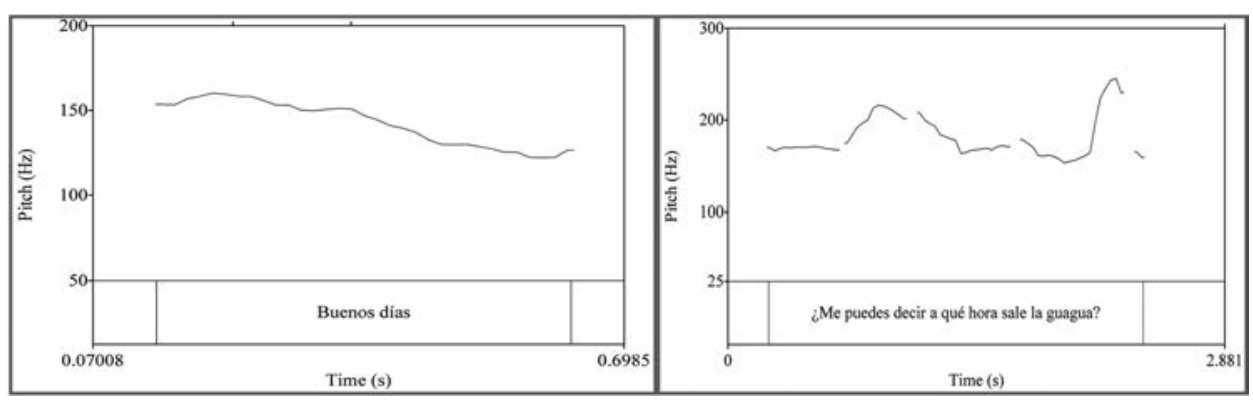

Figuras 3-4. Enunciativa (izquierda) e interrogativa (derecha) obtenidas con el programa Praat (corpus semiespontáneo).

\subsection{Obtención y tratamiento de los datos}

El corpus oral se registró en una grabadora Marantz PMD 222 y un micrófono direccional Shure SM 48 propiedad del Laboratorio de Fonética, Servicio General de Apoyo a la Investigación (SEGAI) de la Universidad de La Laguna. Las grabaciones se realizaron en un entorno cómodo para el informante con el objetivo de posibilitar que los datos tuvieran mayor naturalidad.

La señal analógica grabada se digitalizó con el programa Goldwave (versión 4.25) convirtiendo cada una de las frases en ficheros wave, una vez eliminados los posibles ruidos y optimizada la señal resultante que tiene una frecuencia de muestreo de $18.000 \mathrm{~Hz}$.

11. En nuestro estudio las oraciones breves que tienen un solo pico tonal suponen el $71,1 \%$ y $72 \%$ en las declarativas e interrogativas del corpus inducido, respectivamente, y un 92,8 y $93,3 \%$ en una y otra modalidad en el corpus Map task. Las oraciones largas que presentan más de un pico tonal suponen el $100 \%$ y $82,2 \%$ en las declarativas e interrogativas del primero de estos corpus, respectivamente, y un 100 y $71,4 \%$ en una y otra modalidad en el segundo tipo de corpus. 
Los ficheros wave se etiquetaron según la codificación consensuada en el marco de AMPER que considera para cada oración del corpus ad hoc 9 dígitos + la extensión correspondiente al tipo de fichero (v. gr. WCo1kwki1.wav $\left.{ }^{12}\right)$.

\subsection{Análisis acústico de los datos}

El análisis acústico se llevó a cabo con las rutinas creadas ad hoc en el año 2006 por el grupo AMPER-Astur de la Universidad de Oviedo en el entorno Matlab (versión 7.0.4.) ${ }^{13}$. El análisis tiene diferentes fases y cada frase es pasada por una serie de subrutinas. El punto de partida es el oscilograma de la frase (figura 5) en el que se hace una segmentación de las vocales que se analizan como bloques discretos asignándoles tres valores de $\mathrm{F}_{0}$ (inicio, medio y final), uno de duración y otro de intensidad (figura 6).

Por otro lado, el corpus de contraste (semiespontáneo y espontáneo) se analizó con un script para Praat proporcionado por AMPER Internacional ${ }^{14}$. Se trata de una serie de rutinas creadas para este programa que permiten analizar acústicamente y extraer datos de $\mathrm{F}_{0}$ de una manera más rápida y eficaz que utilizando el método manual. En cuanto a los criterios de segmentación, para el corpus más espontáneo se siguieron los mismos que en el experimental.

12. Los 9 dígitos de la codificación tienen el siguiente significado: $\mathrm{W}$ (dominio lingüístico español); C (variedad español de Canarias); o (isla de La Gomera); número impar (mujer) y número par (hombre): los números 1-2 son para zona urbana sin estudios superiores; los números 3-4 se adjudican a zona rural sin estudios superiores y los números 5-6 a zona urbana con estudios superiores. Los dígitos 5, 6, 7 son letras para identificar cada oración; con el dígito 8 se representa la modalidad: a (declarativa)/i (interrogativa) y, por último, con el dígito 9 se indica el número de repetición de la frase $(1,2$ ó 3). P.ej.: una oración codificada como WCo1kwkil nos da la siguiente información: primera repetición de la oración interrogativa con estructura SN A + SPrep A (sintagma nominal agudo más sintagma preposicional agudo) emitida por una mujer urbana sin estudios superiores de la isla de La Gomera. WCo1: zona urbana sin estudios superiores en voz femenina; WCo2: zona urbana sin estudios superiores en voz masculina; WCo3: zona rural sin estudios superiores en voz femenina. WCo4: zona rural sin estudios superiores en voz masculina. WCo5: zona urbana con estudios superiores en voz femenina. WCo6: zona urbana con estudios superiores en voz masculina.

13. El programa AMPER 2006 del grupo AMPER-Astur parte de otro desarrollado con anterioridad por Antonio Romano (AMPER-fox y AMPER-dat) en el entorno Matlab con el objetivo de agilizar los protocolos de segmentación, estilización y organización de los resultados. Para más información sobre el programa y las subrutinas creadas por el Centre de Dialectologie de Grenoble véase Romano et al. 2005; sobre la adaptación realizada por el grupo Amper-Astur, véase López-Bobo et al. 2007. Licencia de Matlab $n^{\circ} 256105$.

14. Descarga libre del programa en: http://www.fon.hum.uva.nl/praat/. El script fue proporcionado por el Centre de Dialectologie de l'Universitè Stendhal-Grenoble III. 

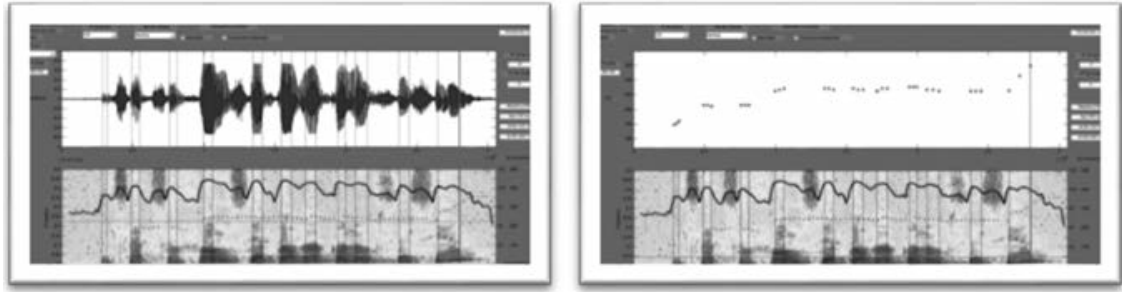

Figuras 5-6. Oscilograma y espectrograma de una señal sonora (derecha)/ Ventana principal que representa las frecuencias fundamentales (izquierda) una vez estilizadas (arriba) y el espectrograma con las zonas delimitadas (debajo).

\subsection{Umbral diferencial}

Para valorar la relevancia de las diferencias tonales consideraremos significativa toda diferencia que alcance o supere el semitono y medio ${ }^{15}$, valor acordado por Rietveld y Gussenhoven hace años (1985) y ratificado por Pamies-Bertrán et al. (2002).

\section{Descripción fonética: declarativas e interrogativas de La Gomera}

\subsection{Corpus experimental}

La media de las oraciones declarativas con finales agudos, llano y esdrújulo de los seis informantes gomeros puede verse en las figuras $7-12^{16}$.

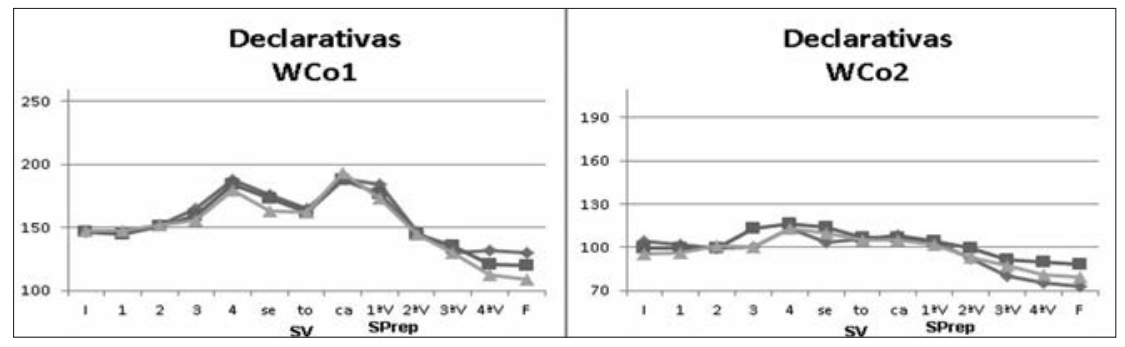

Figuras 7-8. Declarativas de WCo1 y WCo2.

15. El término "significativo" se utiliza en relación con el umbral de percepción de 1,5 St. Por tanto, no debe confundirse con el sentido específico que tiene en el ámbito estadístico.

16. Cada una de las curvas que aparece en los gráficos representa la media de 9 repeticiones, esto es: tres repeticiones de cada una de las frases que se caracterizan por el mismo SV y SPrep pero que se diferencian en el SN. Teniendo en cuenta que el objetivo es el SPrep, las oraciones han sido agrupadas según la tipología acentual del sintagma final. 

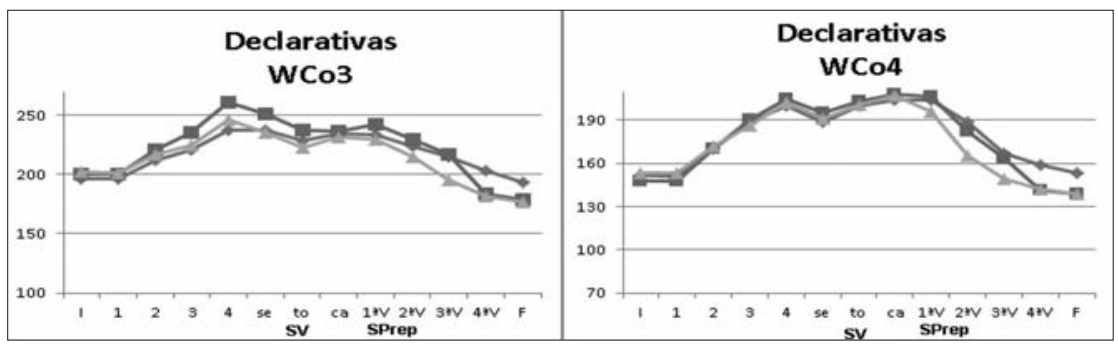

Figuras 9-10. Declarativas de WCo3 y WCo4.
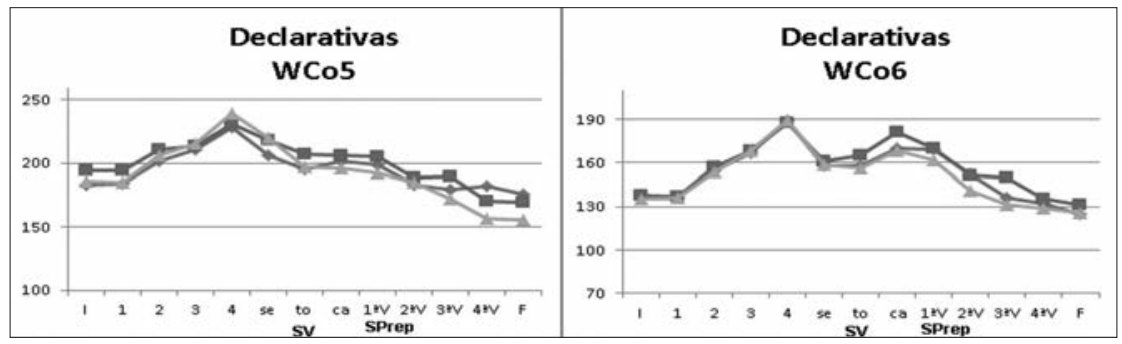

Figuras 11-12. Declarativas de WCo5 y WCo6.

- SPrepE

Como se observa en los gráficos precedentes, el núcleo tonal de las curvas es muy similar en los dos sexos: se caracteriza por una caída tonal desde el $\mathrm{PMx}_{2}{ }^{17}$. que coincide generalmente con un punto de la frontera SV/SPrep, hasta el final absoluto. En la tabla de datos 4 se muestra el valor medio en $\mathrm{Hz}$ del $\mathrm{PMx}_{2}$ o la mayor frecuencia desde donde cae la $\mathrm{F}_{0}$ y su posición en la oración.

Tabla 4. Valor medio (en $\mathrm{Hz}$ ) del PMx2 y su posición en la oración

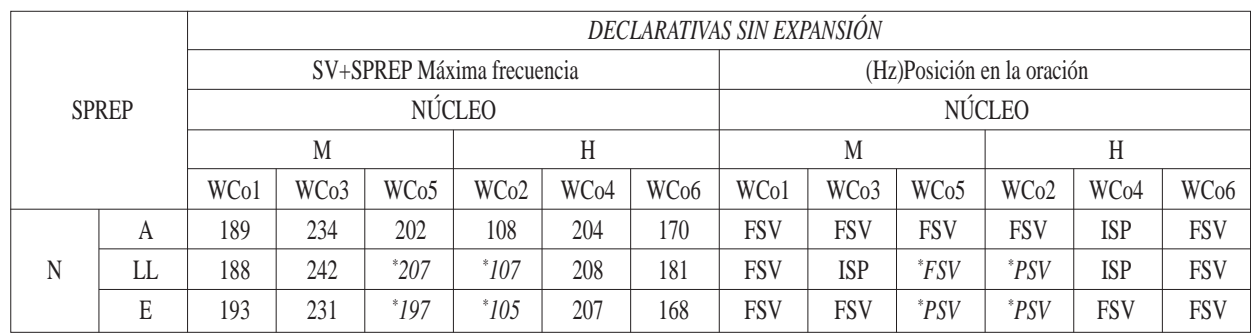

17. Cuando la estructura final es llana o esdrújula en WCo2 (gráfico 8) y WCo5 (gráfico 11) no se puede hablar propiamente de un segundo pico tonal sino más bien de un mantenimiento de la F0 antes 
Sin distinguir entre informantes masculinos y femeninos, si relacionamos el primer pico con el segundo o, cuando este no existe propiamente, con la máxima frecuencia a partir de la cual se da el descenso tonal hasta el final, se observa que en un $66,7 \%$ de los casos el primer pico es más elevado con lo cual se genera una pendiente descendente que solo en un $33,3 \%$ en agudos, $16,7 \%$ en llanos y $50 \%$ en esdrújulos alcanza o supera el umbral perceptivo ${ }^{18}$. Véase la tabla número 5.

Tabla 5. Diferencia (en st) entre el PMx1 y PMx2

\begin{tabular}{|c|c|c|c|c|c|c|c|}
\hline \multirow{4}{*}{\multicolumn{2}{|c|}{ SPREP }} & \multicolumn{6}{|c|}{ DECLARATIVAS SIN EXPANSIÓN } \\
\hline & & \multicolumn{6}{|c|}{ Diferencia (st) entre $\mathrm{PMx}_{1}$ y $\mathrm{PMx}_{2}$ o máxima frecuencia } \\
\hline & & \multicolumn{3}{|c|}{ M } & \multicolumn{3}{|c|}{$\mathrm{H}$} \\
\hline & & WCo1 & WCo3 & WCo5 & WCo2 & WCo4 & WCo6 \\
\hline \multirow{3}{*}{$\mathrm{N}$} & A & 0,1 & $-0,2$ & $-2,1$ & $-0,8$ & 0,3 & $-1,8$ \\
\hline & LL & 0,4 & $-1,3$ & $-1,9$ & $-1,4$ & 0,2 & $-0,6$ \\
\hline & E & 1,2 & $-1,1$ & $-3,3$ & $-1,5$ & 0,4 & -2 \\
\hline
\end{tabular}

Teniendo en cuenta, pues, que la distancia entre los dos picos tonales correspondientes a los dos primeros acentos supera en porcentajes muy bajos las diferencias mínimas perceptibles, no podemos considerar que se produzca mayoritariamente un escalonamiento tonal descendente (downstep). Por tanto, habría que considerar que, en general, se da un mantenimiento de las dos primeras cimas acentuales y sería la tercera, esto es el valor más elevado del tercer acento, la que efectivamente provoca en esta modalidad el declive acusado (Martínez Celdrán 2011).

En cuanto a las interrogativas, tal como se puede ver en las figuras 13-18, coexisten dos patrones entonativos teniendo en cuenta el comportamiento tonal pos-

del descenso final. En las tablas, este valor está representado con *. Por otra parte, en los informantes rurales (WCo3 y WCo4, gráficos 9 y 10) la escasa profundidad del valle anterior (V1-PMx2) en los agudos de la mujer y en los tres acentos del hombre implica que en términos perceptibles no se da realmente un pico tonal puesto que no se alcanza el semitono y medio. Por tanto, registramos en estos casos un sostenimiento de la frecuencia durante el segundo acento.

18. Obsérvese en la tabla 5 que los únicos valores positivos se dan siempre en los informantes WCo1 y WCo4 debido a que el PMx2 se sitúa por encima del PMx1. No obstante, los valores nunca alcanzan el semitono y medio. 
terior al $\mathrm{PMx}_{1}$, esto es, el ascendente -WCo1 y WCo6- y el descendente -WCo2, WCo3, Wco4 y WCo5-.

El patrón descendente en su variante circunfleja (figuras 14-17) se caracteriza por un $\mathrm{PMx}_{2}$ alineado en la sílaba tónica nuclear ${ }^{19}$, esto es, última, penúltima o antepenúltima sílaba según se trate de una estructura aguda, llana o esdrújula. Finalmente desciende en las última/as sílaba/ $\mathrm{as}^{20}$, de manera más acusada en los finales esdrújulos y llanos y menos en los agudos.

El patrón ascendente (figuras 13 y 18) se caracteriza por un descenso desde la cumbre inicial -más brusco que en los anteriores- que llega en la mujer (gráfico 13) hasta la penúltima ${ }^{21}$ o antepenúltima ${ }^{22}$ y en el hombre (gráfico 18) hasta la pretónica del núcleo ${ }^{23}$ para, finalmente, volver a ascender de manera más acusada en las estructuras llanas y esdrújulas y menos en las agudas.

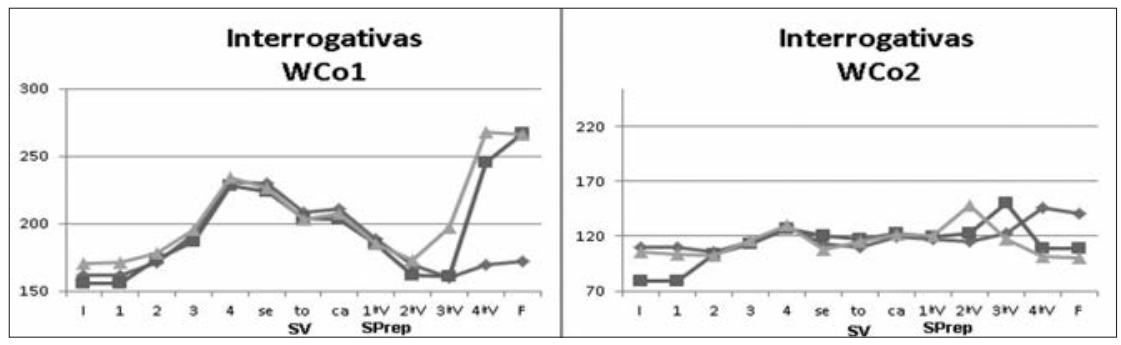

Figuras 13-14. Interrogativas de WCo1 y WCo2.

19. Hay que exceptuar, no obstante, que en WCo3 si bien la frecuencia más elevada culmina en la tónica de los esdrújulos, la diferencia respecto de la vocal posterior es insignificante $(279-277 \mathrm{~Hz})$ por lo que forma, más que un pico, una meseta tonal. En los llanos de WCo5 (gráfico 17) el pico se pospone y, por ello, no hay alineamiento tonal.

20. Las rutinas de Matlab solo miden la F0 en tres puntos de los núcleos silábicos esto es inicial, medio y final. Por tanto, cuando hablamos del final de la F0 nos referimos al último punto que se mide en la vocal por lo que, si la última sílaba está trabada por una consonante sonora, la F0 continuará en esta consonante su trayectoria ascendente o descendente, según sea el caso, como sucede fundamentalmente con la palabra "saxofón" en este tipo de oraciones.

21. Se trata de la tónica en LL y átona en A (10 $10^{a}$ sílaba). En la mujer sin estudios de zona urbana el descenso desde el PMx1 no es progresivo sino que la frecuencia disminuye hasta el FSV y desde esa posición se da el declive más acusado.

22. Tónica en E ( $9^{\mathrm{a}}$ sílaba).

23. $10^{\text {a }}, 9^{\mathrm{a}}$ y $8^{\mathrm{a}}$ sílaba, en A, LL y E, respectivamente. Si bien el movimiento de descenso llega hasta las posiciones indicadas, en términos significativos no hay diferencias relevantes entre la pretónica y la tónica en los esdrújulos. 


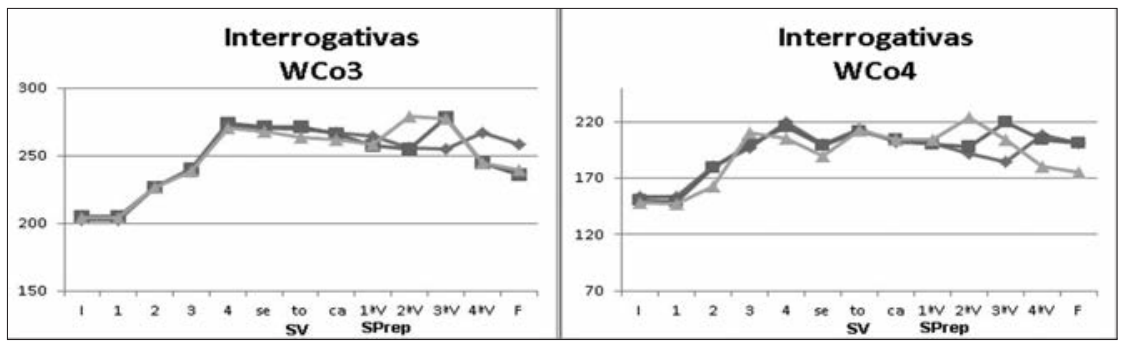

Figuras 15-16. Interrogativas de WCo3 y WCo4.

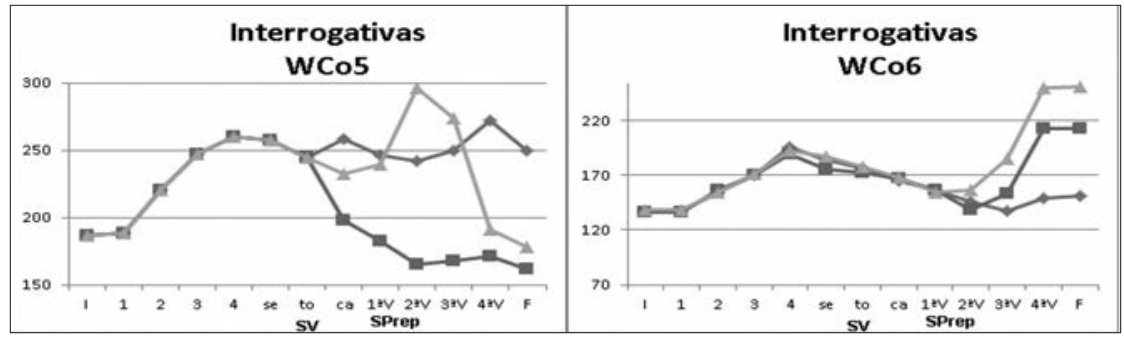

Figuras 17-18. Interrogativas de WCo5 y WCo6.

$\neg$ SPrepA $\rightarrow$-SPrepLL $\triangle$-SPrepE

En la siguiente tabla de datos puede verse la frecuencia que alcanza el $\mathrm{PMx}_{2}$ en los finales circunflejos y la frecuencia máxima de los finales ascendentes.

Tabla 6. Valor medio (en Hz) del PMx2 y su posición en la oración

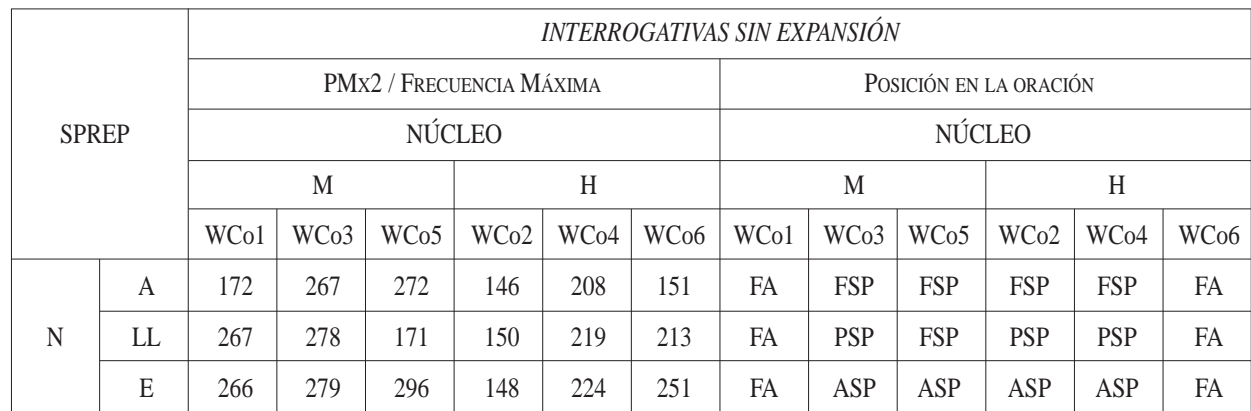

De este modo, la frecuencia del pico del último acento en los contornos circunflejos supera la del pico inicial -en un 25\%, $75 \%$ y $100 \%$ en A, LL y E, respectivamente, de manera que la pendiente que origina la secuencia es ascen- 
dente ${ }^{24}$. No obstante, si tenemos en cuenta los valores que rebasan el umbral diferencial (tabla 7), los porcentajes son más escasos $-25 \%$ en A y LL y $50 \%$ en E-. En definitiva, si bien se observa que el PMx2 tiende a ascender en términos relativos, la línea que genera respecto del pico inicial sería, de manera general, nivelada.

En los contornos ascendentes la frecuencia máxima situada al final de las oraciones supera la de la cumbre inicial de manera significativa si la estructura es llana o esdrújula; si es aguda, en cambio, la pendiente que se genera es siempre descendente. En la tabla 7 pueden verse las diferencias relativas entre el primer pico y el segundo, en el caso de los finales circunflejos, y entre el primero y la frecuencia máxima nuclear en el caso de los finales ascendentes.

Tabla 7. Diferencia (en st) entre el PMx1 y PMx2

\begin{tabular}{|c|c|c|c|c|c|c|c|}
\hline & & INT & $O G A T I$ & SIN EXI & $I O ́ N$ & & \\
\hline & & & ncia (st) & $\mathrm{PMx}_{1}$ & $\mathrm{Mx}_{2}$ & & \\
\hline & & & M & & & $\mathrm{H}$ & \\
\hline PMx1 & & WCo1 & WCo3 & WCo5 & WCo 2 & WCo4 & WCo6 \\
\hline & A & -5 & $-0,2$ & $-0,8$ & 3,2 & $-0,9$ & $-4,5$ \\
\hline $\mathrm{N}$ & LL & 2,7 & 0,2 & $-7,2$ & 2,9 & 0,3 & 2 \\
\hline & E & 2,2 & 0,6 & 2,2 & 2,2 & 1,1 & 4,5 \\
\hline
\end{tabular}

En definitiva, en las interrogativas encontramos dos tipos de tonema si bien el predominante en la isla es el ascendente-descendente, esto es, el llamado patrón circunflejo. La entonación final ascendente es, por tanto, minoritaria. Los dos patrones interrogativos se asemejan en el hecho de presentar un ascenso en el tramo final y eso es lo que marca el carácter interrogativo -frente a la otra modalidad oracional-. No obstante, si relacionamos los dos máximos tonales de la interrogativa, podemos concluir que solo se da un ascenso entre estos si las es-

24. Con la excepción de los llanos de WCo5 en los que el pico nuclear, muy poco pronunciado se da en frecuencias muy bajas hasta el punto de que el contorno tonal parece más propio de una declarativa que de una interrogativa. En esta modalidad oracional nos interesa ver si la frecuencia alcanzada por el tonema es la más elevada de toda la frase o, por el contrario, se mantiene en términos relativos al mismo nivel que el pico inicial. 
tructuras finales son llanas y esdrújulas y el contorno tonemático ascendente, pero no en las agudas en que esto es infrecuente. Si el contorno tonemático es ascendente-descendente las escasas diferencias relativas entre las dos cumbres máximas de las oraciones, evidencian el mantenimiento de las frecuencias y no el ascenso de una sobre la otra. Al centrarnos en el último sintagma se comprueba, por tanto, que en el núcleo entonativo se dan diferencias entre las dos modalidades oracionales puesto que contrasta el descenso de declarativas frente al ascenso de interrogativas (ascenso-descenso o ascenso) cumpliendo una función distintiva.

\subsection{Corpus de contraste}

Mostraremos a continuación el comportamiento en las oraciones declarativas e interrogativas en los dos tipos de corpus analizados (inducido y map task) siguiendo la clasificación esbozada en el apartado de Metodología, esto es, separando las oraciones con un solo pico (1PMx) de las que tienen mayor número de cumbres tonales $(+1 \mathrm{PMx})^{25}$. Los gráficos de líneas ilustrarán la frecuencia media en el sexo femenino y masculino de algunos puntos relevantes de las curvas, esto es, inicio absoluto (I), pretónica (pre), tónica (to) y postónica (pos, en palabras llanas) del acento nuclear y final absoluto (F). Ello nos permitirá caracterizar el patrón entonativo a partir de la dirección del tonema en los dos estilos de habla.

\subsubsection{Comportamiento en las oraciones con un PMx}

Las figuras 19 y 20 ilustran las enunciativas según el tipo de acento final (llano/agudo ${ }^{26}$ ) en voz femenina y masculina; como puede verse, se caracterizan por un tonema final descendente en los dos tipos de corpus ${ }^{27}$.

25. Como se indicó en el apartado de la Metodología las oraciones con un solo pico tonal máximo tienen generalmente una extensión de 7 sílabas en las declarativas y de 6 en las interrogativas. Las oraciones que tienen más de una cumbre tonal máxima superan dichas cifras: +7 sílabas en declarativas y +6 sílabas en interrogativas.

26. En los finales agudos, al carecer de postónica, se ha repetido en los gráficos el valor final absoluto.

27. Cuando la oración tiene un solo acento la frecuencia más elevada de la oración, esto es, su máximo tonal, se da en su tónica o en su pretónica esto es, $2,7 \%$ en el CS y 15,4\% en el CE para cada posición, respectivamente. 


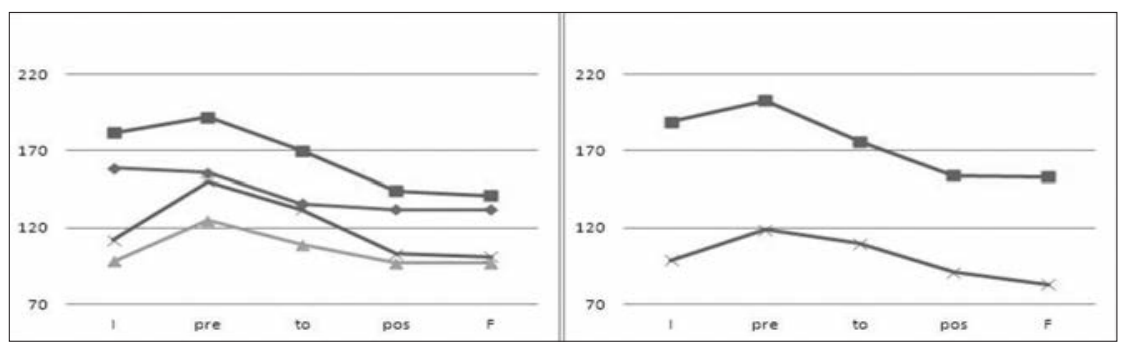

Figura 19. Corpus semiespontáneo.

Figura 20. Corpus espontáneo.

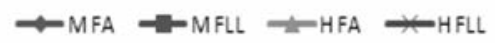

En las interrogativas, en cambio, el promedio de $\mathrm{F}_{0}$ revela que el núcleo final se caracteriza por un movimiento tonal circunflejo (ascendente-descendente) que llega, incluso, a abarcar toda la frase cuando el número de acentos es muy reducido. No obstante, en los finales agudos, al ser la tónica la última de la frase y carecer de postónica -como puede verse en las figuras 21-22- el descenso de la frecuencia fundamental se trunca o es imperceptible.

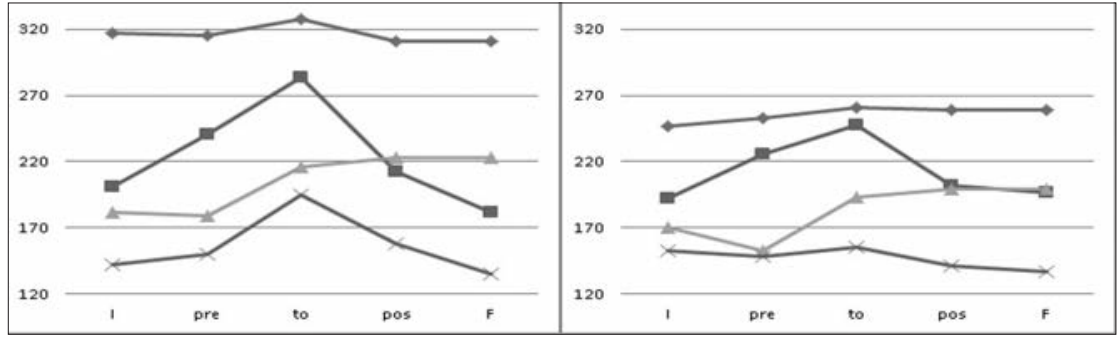

Figura 21. Corpus semiespontáneo.

Figura 22. Corpus espontáneo.

$\leadsto$ MFA - MFLL $\simeq$ HFA $\nrightarrow$ HFLL

\subsubsection{Comportamiento en las oraciones con más de un PMx}

En las declarativas (figuras 23-24), el pico que marca el descenso final se sitúa frecuentemente en el penúltimo acento de la oración. Desde esta segunda elevación del tono, por tanto, la $\mathrm{F}_{0}$ sigue la declinación normal de esta modalidad, quedando el último acento, como puede verse en las siguientes ilustraciones promediadas, en una frecuencia baja. 


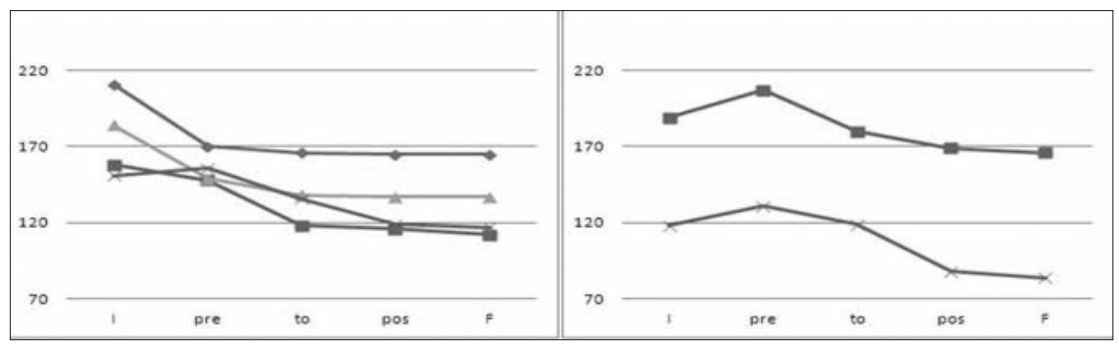

Figura 23. Corpus semiespontáneo.

Figura 24. Corpus espontáneo.

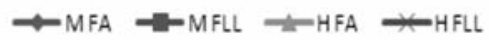

Las interrogativas, en cambio (figuras 25-26), se caracterizan en el núcleo tonal por el patrón circunflejo que hemos visto mayoritariamente en el corpus experimental; en los finales agudos, como puede verse en los gráficos 25 y 26 , el último descenso se suspende o es muy breve por carecer de segmento postónico.

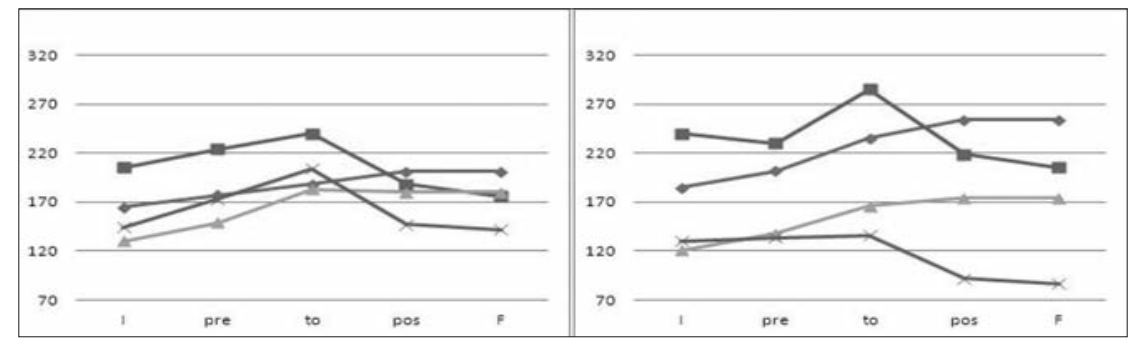

Figura 25. Corpus semiespontáneo.

Figura 26. Corpus espontáneo.

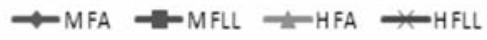

Finalmente, si relacionamos el $\mathrm{PMx}_{2}$ con el $\mathrm{PMx}_{1}$ en las secuencias sin promediar, el resultado es el siguiente:

$1^{\circ}$ En las declarativas, el segundo pico tonal se sitúa por debajo del primero en el $50 \%$ (CS) y $100 \%$ (CE) de los $\operatorname{casos}^{28}$. Sin embargo, solo podemos hablar de escalonamiento tonal descendente de los picos en un $14,3 \%$ y

28. Los datos se han estudiado teniendo en cuenta la estructura acentual de la palabra en la que recae el último pico máximo que es en todos los casos llana. 
$50 \%$, respectivamente si consideramos los casos en que la pendiente descendente generada alcanza o supera el semitono y medio.

$2^{\circ}$ En las interrogativas, la segunda cima tonal supera la frecuencia de la primera en un $57,9 \%$ y $69 \%$ en palabras agudas y llanas del CS, respectivamente; en el CE en un $60 \%$ y 66,7\% en los mismos tipos de acentos. Ahora bien, la diferencia entre ambas cumbres roza o supera el umbral perceptivo y, por tanto, se suspende la declinación de los picos, dando lugar al escalonamiento tonal ascendente de los mismos en un 52,6\% (A) y 37,9\% (LL) de los casos de CS y de en un 30\% (A) y $0 \%$ (LL) en el CE.

En definitiva, el estudio de las declarativas en un corpus de análisis de contraste de tipo semiespontáneo y espontáneo evidencia que, con independencia del tipo de estilo de habla que se analice o el mayor o menor relieve tonal, esta modalidad se caracteriza por presentar de manera general un descenso final durante el núcleo entonativo; cuando las enunciativas se reducen a un acento puede alinearse en este el único pico de la oración. En relación con la línea melódica que describen los máximos tonales inicial y final de las declarativas con mayor relieve tonal, considerando que la distancia solo supera el semitono y medio en reducidos casos, concluimos que es nivelada y no descendente.

Las interrogativas, por su parte, presentan el patrón más general en el archipiélago canario, esto es, un movimiento ascendente-descendente vinculado al acento nuclear. Cuando la oración reduce el número de acentos el movimiento abarca toda la frase. En función de la estructura acentual final, hemos visto que el patrón se manifiesta con truncamiento del descenso en las estructuras agudas y sin truncamiento en las llanas. Considerando los máximos tonales inicial y final, la línea melódica que resulta no es ascendente sino generalmente nivelada, como en la otra modalidad oracional.

\section{Discusión de los resultados}

En los corpus de estudio que hemos cotejado hemos encontrado las siguientes oposiciones tonemáticas para distinguir la modalidad declarativa de la interrogativa:

\section{$1^{\mathrm{a}}$ Ascenso en interrogativas/descenso en declarativas}

Esta oposición tonemática se da solo en el corpus experimental del habla urbana de La Gomera (mujer sin estudios -WCo1- hombre con estudios -WCo6-). La misma oposición fue registrada por Dorta (2013) en habla urbana sin estudios de La Gomera y por Hernández et al. (2014) en habla rural de El Hierro y en habla 
rural de La Palma. Asimismo, se ha registrado en los dialectos peninsulares y americanos (Garrido 1991; Quilis 1993 o Sosa 1999).

En trabajos realizados en el marco de AMPER, diversos estudios recientes confirman la oposición tonemática en el ámbito del español peninsular (Madrid, Ramírez Verdugo 2005; Donostia y Bilbao [País Vasco], Elejabietia et al. 2008; Sevilla [Andalucía], Congosto y et al. 2008; Santillana del Mar [Cantabria], Viejo Lucio-Villegas 2008; Salamanca y Valladolid [Castilla y León], Zamora Salamanca et al. 2007; y Zaragoza y Jaca [Aragón], González Olivera et al. 2007 y Simón Casas 2007) y, más esporádicamente, en el español americano (Santiago de Chile, Román et al. 2008; Montero Santa Cruz [Bolivia], Congosto 2007).

\section{$2^{\text {a }}$ Ascenso-descenso en interrogativas/descenso en declarativas}

Esta oposición se registró mayoritariamente en el corpus experimental analizado en el presente estudio, tanto en habla urbana como rural (WCo2, WCo3, WCo4, WCo5), y fue la única que se encontró en el corpus de contraste. Asimismo, es la que se ha registrado generalmente en las islas Canarias (v. gr. Dorta 2013 y Hernández et al. 2014) y relaciona la variedad canaria con el español de América (v. gr. García Riverón 1996: 79; Sosa 1999: 212-213). Así se ha confirmado en estudios recientes realizados en el marco de AMPER puesto que se ha registrado en Buenos Aires (Argentina), Gurlekian y Toledo 2008; Andes venezolanos, Díaz y Rojas 2012 y Dorta Ed. 2013; La Habana, Santiago de Cuba y Santa Clara (Cuba), García Riverón y Fernández Pérez Terán 2007, Dorta Ed. 2013 y Dorta et al. 2015; y Medellín (Colombia), Díaz et al. 2015 (en prensa).

\section{Conclusiones}

$1^{\text {a }}$ En el corpus experimental hemos registrado dos tipos de oposiciones tonemáticas mientras que en el de contraste solo una (tabla 8).

Tabla 8. Oposiciones fonemáticas

\begin{tabular}{|l|l|}
\hline \multicolumn{3}{|c|}{ ESQUEMAS ACÚSTICOS } \\
\hline Tipo de corpus & DECLARATIVAS \\
\hline Corpus experimental & \\
\hline Corpus de contrastes & \\
\hline
\end{tabular}


$2^{\text {a }}$ En las declarativas la mayor o menor espontaneidad del corpus no interfiere sustancialmente en el patrón obtenido. En cambio, este factor sí incide claramente en el patrón nuclear de las interrogativas no pronominales pues en algunos informantes (WCo1 y WCo6) el corpus experimental, más formal, ha determinado el uso de un patrón ascendente en lugar del circunflejo más general y el único registrado en el corpus más espontáneo.

$3^{\text {a }}$ De la conclusión anterior se deriva la confirmación de nuestra hipótesis de partida, esto es, el estudio del corpus de contraste ha evidenciado que en la isla de La Gomera se da una única oposición entonativa, esto es, descenso/ascensodescenso, en declarativas e interrogativas, respectivamente (común en Canarias) por lo que la manifestación de la otra en ambas modalidades, esto es, descenso/ascenso queda relegada a aquellos contextos que implican mayor formalidad en los que el hablante imita la configuración del castellano septentrional y, por tanto, su existencia no constituye un rasgo conservador de la isla.

\section{Bibliografía}

ALMEIDA, M. y DÍAZ ALAYÓN, C. (1988). El español de Canarias. Santa Cruz de Tenerife.

ALVAR, M. (1968). Estudios canarios I. Las Palmas de Gran Canaria: Cabildo Insular de Gran Canaria.

CONGOSTO MARTÍN, Y. (2007). "Dialectología de la entonación. Interrogativas absolutas en el español de España (Sevilla, Badajoz) y en el español de América (Bolivia)" en La prosodia en el ámbito lingüístico románico (Ed. J. Dorta). Santa Cruz de Tenerife: La Página Ediciones (Colección Universidad): 389-415.

CONGOSTO MARTÍN, Y. et al. (2008). "Picos tonales, acento léxico y límites sintagmáticos en enunciados declarativos e interrogativos absolutos sin expansión en el objeto vs. con expansión (en el sujeto y en el objeto)". Language Design. Journal of Theoretical and Experimental Linguistics (Special Issue 2): 203-212.

CORRALES ZUMBADO, C. et al. (1998). El español de Canarias: Guía bibliográfica. La Laguna: Instituto de Estudios Canarios.

CORRALES, C. y CORBELLA, D. (2004). "Primeros testimonios e impresiones sobre el habla canaria". Anuario de Estudios Atlánticos 50 (1): 71-120.

DÍAZ, CH. (2013). Contribución al Atlas Prosódico de Canarias (AMPERCan): Declarativas e interrogativas de La Gomera (Islas Canarias). Tesis doctoral. Santa Cruz de Tenerife: Universidad de La Laguna. 
DÍAZ, CH. y ROJAS, N. (2012). "Declarativas e interrogativas sin expansión en voz femenina urbana: Islas Canarias vs. Distrito capital (Caracas)" en Workshop internacional. Entonación hispánica: variantes americanas y españolas 1: 11-13 de abril de 2012. Santa Cruz de Tenerife: Laboratorio de Fonética, Universidad de La Laguna.

DÍAZ, CH. et al. (2015 en prensa). "Intonation across Two Border Areas in the North Andean Region: Mérida (Venezuela) and Medellin (Colombia)" en Issues in Hispanic and Lusophone Linguistics. John Benjamins.

DORTA, J. (2000). "Entonación Hispánica: No pronominales vs. Pronominales". Lingüística Española Actual 22 (1): 51-76.

DORTA, J. (2007). "La entonación de la interrogación no pronominal en zonas rurales de Canarias: Gran Canaria y La Gomera" en Actas del VI Congreso de Lingüística General T II. Madrid: Arco/Libros: 1915-1929.

DORTA, J. (2008). "La entonación de las interrogativas simples en voz femenina. Zonas urbanas de las islas canarias" en La variation diathopique de l'intonation dans le domain roumain et roman. Rumanía: Presses de l'Université «Al. 1. Cuza» de Ia i : 123-150.

DORTA, J. (2013). "Estudio fonético-fonológico de la entonación declarativa e interrogativa canaria en voz femenina" en De lingüística, traducción y léxicofraseología: Homenaje a Juan de Dios Luque Durán (Ed. A. Pamies). Granada: Editorial Comares: 173-197.

DORTA, J. (Ed.) (2013). Estudio comparativo preliminar de la entonación de Canarias, Cuba y Venezuela. Madrid-Tenerife: La Página ediciones (Colección Universidad).

DORTA, J. y DÍAZ, CH. (2014). "Reconocimiento perceptivo de patrones interrogativos coexistentes en Canarias". Fortunatae 25, Homenaje al Dr. Fremiot Hernández González: 115-128.

DORTA, J. et al. (2015). "Continuidad prosódica en habla experimental y espontánea de Canarias y Cuba: variación y rango tonal en las interrogativas no pronominales en Les variations diasystématiques et leurs interdépendances dans les langues romanes. (Eds. K. Jeppesen Kragh y J. Lindschouw; Collection dirigée par E. Casanova [València], J.-P. Chauveau [Nancy, ATILF] y Hans Goebl [Salzbourg]). Strasbourg: Ouvrage publié avec l'appui de l'Académie Royale des Sciences et Belles-lettres de Danemark et du Lektor Knud Henders Legatfond: 145-159.

ELEJABIETIA, A. et al. (2008). "La prosodia del castellano en tres ciudades vascas: oraciones con y sin expansión en el SN". Language Design. Journal of Theoretical and Experimental Linguistic (Special Issue 2): 147-154. 
GARCÍA RIVERÓN, R. (1996). Aspectos de la entonación hispánica II. Análisis acústico de muestras del español de Cuba. Cáceres: Universidad de Extremadura.

GARCÍA RIVERÓN, R. y FERNÁNDEZ PÉREZ TERÁN, F. (2007). “AMPERCUBA: primeros resultados en un proyecto conjunto" en Actas del III Congreso de Fonética Experimental. Santiago: Universidad de Santiago.

GARRIDO ALMIÑANA, J. M. (1991). Modelización de patrones melódicos del español para la síntesis y el reconocimiento de habla. Barcelona: Departamento de Filología Española, Universitat Autònoma de Barcelona.

GONZÁLEZ OLIVERA, M. P. et al. (2007). "Estudio comparativo de la prosodia de Zaragoza y Jaca” en La prosodia en el ámbito lingüístico románico (Ed. J. Dorta). Santa Cruz de Tenerife: La Página Ediciones (Colección Universidad): 225-244.

GURLEKIAN, J. y TOLEDO, G. (2008). "Datos preliminares del Amper-Argentina: las oraciones declarativas e interrogativas absolutas sin expansión”. Language Design. Journal of Theoretical and Experimental Linguistics (Special Issue 2): 213-220.

HERNÁNDEZ, B. (2007). "La entonación de las declarativas simples en zonas rurales de Canarias: La Gomera y Gran Canaria" en Actas del VI Congreso de Lingüística General. Madrid: Arco/Libros: 63-79.

HERNÁNDEZ DÍAZ, B. et al. (2011). "La entonación de las declarativas simples en voz femenina. Zonas urbanas de las islas canarias" en El estudio de la prosodia en España en el S. XXI. Perspectivas y Ámbitos (Eds. A. Hidalgo, Y. Congosto y M. Quilis). Valencia: Universitat de València: 109-124.

HERNÁNDEZ, B. et al. (2014). "Declarativas e interrogativas en zonas rurales de Canarias" en Fonética Experimental, Educación Superior e Investigación (Eds. Y. Congosto Martín, M. L. Montero Curiel y A. Salvador Plans) T. III. Madrid: Arco Libros: 245-265.

LÓPEZ BOBO, M. J. et al. (2007). “Análisis y representación de la entonación. Replanteamiento metodológico en el marco del proyecto AMPER" en La prosodia en el ámbito lingüístico románico (Ed. J. Dorta). Santa Cruz de Tenerife: La Página Ediciones (Colección Universidad): 17-34.

MARTÍNEZ CELDRÁN, E. (2011). "La línea melódica de la entonación declarativa e interrogativa absoluta en el español de España" en El estudio de la prosodia en España en el S. XXI. Perspectivas y Ámbitos (Eds. A. Hidalgo, Y. Congosto y M. Quilis). Valencia: Universitat de València: 125-140.

MORERA, M. (2003-2004). "Tradición y novedad en el léxico de La Gomera (Canarias)" en Memoriam Manuel Alvar (1923-2001) (Eds. Rosa M. ${ }^{\text {a Casta- }}$ 
ñer y José M. ${ }^{a}$ Enguita) T.II. Zaragoza: Institución "Fernando el Católico": 1525-1534.

PAMIES-BERTRÁN, A. et al. (2002). "Umbrales tonales en español peninsular" en Actas del II Congreso de Fonética Experimental (Ed. J. Díaz García). Sevilla: Universidad de Sevilla: 272-278.

QUILIS, A. (1993). Tratado de fonología y fonética españolas. Madrid: Gredos. RAMÍREZ VERDUGO, M. D. (2005). “Aproximación a la prosodia del habla de Madrid”. Estudios de Fonética Experimental 14: 309-326.

ROMÁN MONTES DE OCA D. et al. (2008). "Rasgos prosódicos de oraciones sin expansión, del español de Santiago de Chile en habla femenina". Language Design. Journal of Theoretical and Experimental Linguistics (Special Issue 2): 137-146.

ROMANO, A. et al. (2005). "La méthodologie AMPER”. Géolinguistique Hors Serie 3: 1-5.

RIETVELD, A. y GUSSENHOVEN, C. (1985). "On the relation between pitch excursion size and prominence". Journal of Phonetics 13: 299-308.

SALVADOR CAJA, G. (1990): "Las hablas canarias" en Actas del Congreso de la Sociedad Española de Lingüística. XX Aniversario. Madrid: Gredos.

SIMÓN CASAS, F. J. (2007). “Análisis de la entonación de los enunciados declarativos e interrogativos sin expansión en un hablante masculino de Jaca (Huesca)" en Actas del III Congreso de Fonética Experimental. Santiago: Universidad de Santiago: 561-567.

SOSA, J.M. (1999). La entonación del español, su estructura fónica, variabilidad y dialectología. Madrid: Cátedra.

TORRES ÁLVAREZ, M.I. (2000). "Oraciones cortas vs. Oraciones largas: diferencias en el patrón entonativo". Revista de Filología de la Universidad de La Laguna 18: 383-397.

TRUJILLO, R. (1978). El silbo gomero: análisis lingüístico. Santa Cruz de Tenerife: Editorial Interinsular Canaria-Instituto de Lingüística "Andrés Bello".

VIEJO LUCIO-VILLEGAS M. (2008). "Primera aproximación a la prosodia de la zona central de Cantabria: enunciados con estructura sujeto-verbo-objeto". Language Design. Journal of Theoretical and Experimental Linguistics (Special Issue 2): 171-178.

ZAMORA SALAMANCA, F. J. et al. (2007). “Aproximación a la prosodia del habla urbana de Salamanca (en contraste con la de Valladolid)" en La prosodia en el ámbito lingüístico románico (Ed. J. Dorta). Santa Cruz de Tenerife: La Página Ediciones (Colección Universidad): 179-202. 